\title{
CONTRIBUIC̣ÃO DAS BEBIDAS PARA A HIDRATAÇÃO, ANTES, DURANTE E DEPOIS DA ACTIVIDADE FÍSICA EM ESTUDANTES UNIVERSITÁRIOS
}

\author{
CONTRIBUTION BEVERAGES FOR HYDRATION BEFORE, \\ DURING AND AFTER PHYSICAL ACTIVITY IN UNIVERSITY STUDENTS
}

\author{
AUTORES \\ Carla Andreia Lima Ferreira' \\ José Augusto Rodrigues dos Santos ${ }^{2}$ \\ Luiza Kent-Smith ${ }^{3}$ \\ Inês Sofia Morales Salcedo ${ }^{3}$ \\ Nutricionista, Bolseira do Instituto \\ de Bebidas e Saúde \\ ${ }^{2}$ Faculdade Ciências do Desporto e \\ Educação Física da Universidade do Porto \\ ${ }^{3}$ Faculdade Ciências da Nutrição e \\ Alimentação da Universidade do Porto
}

CONTRIBUIC̄̃̃O DAS BEBIDAS

PARA A HIDRATAÇÃO,

ANTES, DURANTE E DEPOIS

DA ACTIVIDADE FÍSICA EM ESTUDANTES UNIVERSITÁRIOS 4(1): 27-38

\section{PALAVRAS-CHAVE}

hidratação; actividade física; bebidas

\section{KEYWORDS}

hydration; physical activity; beverages

data de submissão Maío 2006

data de aceitação Outubro 2007
RESUMO

Introdução: 0 consumo de líquidos antes, durante e depois da actividade física tem efeito benéfico na hidratação corporal de indivíduos fisicamente activos. Previne a desidratação e aumenta a capacidade de desempenho físico. Objectivos: Avaliar os conhecimentos, contributos e o consumo de bebidas antes, durante e depois da actividade física em universitários fisicamente activos, e a composição corporal, actividade física e gasto energético. Metodologia: Foram avaliados 348 alunos, 140 do sexo feminino e 208 do masculino que frequentam do $1^{\circ}$ ao $4^{\circ}$ ano da Licenciatura de Ciências do Desporto e Educação Física da Faculdade de Desporto da Universidade do Porto (FCDEF-UP). Foi utilizado um inquérito específico e parâmetros antropométricos. 0 tratamento estatístico foi feito pelo programa SPSS, versão 13.0 para o Windows e Microsoft Excel 2003 com $p<0,05$. Resultados: Verificou-se que os alunos do $1^{\circ} \mathrm{e}$ 2 ano praticam e gastam mais calorias com actividade física numa semana típica. Cerca de $95 \%$ dos alunos considera necessário o consumo de bebidas antes, durante e depois da actividade física. Discussão: A hidratação é justificativa da necessidade do consumo de bebidas antes, durante e depois da actividade física. Cerca de $85 \%$ consomem bebidas em pelo menos uma das modalidades desportivas praticadas, antes, durante e depois da actividade física. A água foi a bebida mais consumida antes, durante e depois da actividade física curricular e extracurricular.
ABSTRACT

Introduction: intake of liquids before, during and after physical activity has beneficial effects on body hydration of physically active individuals, preventing dehydration and increasing the physical capacity. Objectives: to evaluate the knowledge, contribution and consumption beverages before, during and after physical activity in a group of physically active university students, to evaluate the body composition, physical activity and energy expenditure during weekly physical activity. Methodology: we carried out a survey of 348 students, 140 female and 208 male, currently attending from the 1st to the 4th year of the Faculty of Sport of the University of Porto. They were appraised through a specific questionnaire and anthropometrical evaluation. Statistical treatment was performed using SPSS, version 13.0 for Windows and Microsoft Excel 2003 for $p<0.05$. Results: It was verified that 1 st and 2 nd year students practice and spend more calories with physical activity in a typical week. About $95 \%$ of the students consider it necessary to consume beverages before, during and after physical activity. Hydration is mentioned more often by students as justification for the need and consumption of beverages, before, during and after physical activity. Of the students that practiced physical activity, about $85 \%$ consume beverages before, during and after physical activity, in at least one sport modality practiced. Discussion: Hydration is for the surveyed students the main justification for the need and consumption of beverages before, during and after physical activity. Water is the students' preferred beverage for consumption during physical activity. 


\section{INTRODUC̣ÃO}

A posição do American College of Sports Medicine ${ }^{1}$ fornece uma orientação com respeito à reposição de líquidos e electrólitos. Recomenda a reposição adequada de líquidos para a manutenção da hidratação na promoção de saúde, segurança e óptimo desempenho no decurso da actividade física. ${ }^{17}$

Uma hidratação adequada antes da actividade física é essencial para preservar todas as funções fisiológicas. Indivíduos que ingeriram líquidos antes da actividade física apresentaram uma temperatura interna menor e uma menor frequência cardíaca, durante a actividade física, comparativamente com aqueles que não ingeriram líquidos. Um défice hídrico antes da actividade física pode potencialmente comprometer a regulação térmica e produzir uma sobrecarga cardiovascular durante o esforço. Não existe evidência suficiente para apoiar a hiperhidratação antes da actividade física como um meio para manter a hidratação durante o mesmo. Durante a actividade física, o objectivo da ingestão de líquidos deve ser de equilibrar os líquidos perdidos pelo suor ou quando as taxas de suor são muito altas, repor quanto antes o equilíbrio hídrico. A quantidade e frequência necessárias devem ser ajustadas de acordo com as taxas de suor individual e a tolerância à ingestão de líquidos. IFSM ${ }^{7}$ demonstraram que a ingestão de grandes volumes de líquidos está associada ao maior débito cardíaco, maior fluxo sanguíneo periférico, menor temperatura interna e menor percepção de fadiga durante o exercício. IFSM ${ }^{7}$ reafirmam que a desidratação, mesmo ligeira, pode reduzir o desempenho desportivo. A manutenção da saúde e desempenho desportivo deve justificar a rápida reposição hídrica nos desportistas. A reposição da água e o equilíbrio electrolítico são essenciais para o processo de recuperação após actividade física que resulta das perdas pelo suor. Uma hidratação adequada depois de um programa de actividade física significa começar bem hidratado o próximo treino ${ }^{1,17}$.

Segundo as recomendações da ACSM $^{1}$, a hidratação só é mantida em pessoas fisicamente activas se beberem líquidos suficientes antes, durante e depois de actividade física $^{1,7}$. A análise fiável da actividade física em indivíduos fisicamente activos requer instrumentos específicos de avaliação.

A avaliação da actividade física por questionários é a forma mais habitual utilizada em estudos epidemiológicos. Os questionários têm sido usados em função da aplicabilidade para grandes grupos, de baixo custo e por permitir a recolha de informação precisa sobre o tipo de actividade física e o contexto em que acontecem. Uma análise significativa deve incluir as várias dimensões da actividade física: frequência, duração, intensidade e tipo de exercício, possibilitando uma estimativa do gasto calórico em actividades moderadas ou intensas ${ }^{2,14}$.

O International Physical Activity Questionnaire (IPAQ) foi desenvolvido para servir como um instrumento válido para comparação dos níveis de actividade física entre diversas populações e contextos culturais e sociais. 0 Instituto Holandês de Saúde Publica e Ambiente desenvolveu a Short Questionnaire to Assess Health - enhancing physical activity (SQUASH) válido e reprodutível, curto e com questões sobre actividades habituais e de aplicação simples em vários tipos de populações $^{12,22}$.

$A$ actividade física pode influenciar a composição corporal dando origem a variações responsáveis por alterações das características dos compartimentos corporais. A composição corporal é definida em 4 compartimentos: gordura, água, massa proteica e massa óssea. $\mathrm{Na}$ prática, baseia-se em 2 compartimentos: a massa gorda e a massa magra. 0 termo massa gorda é geralmente designado como tecido adiposo e massa magra o resto do corpo. A avaliação antropométrica da massa gorda a partir das pregas de adiposidade subcutâneas (tricipital, bicipital, subescapular e suprailíaca) é baseada em dois princípios: os locais de medida escolhidos são representativos do tecido adiposo subcutâneo e da relação constante entre a deposição de gordura subcutânea e a gordura perivisceral. A área muscular do braço é estimada a partir dos valores do perímetro médio do braço e prega tricipital ${ }^{6,10,16}$.

o Índice de Massa Corporal (peso/ /estatura) e a razão entre o perímetro da cintura e anca (ICA) são normalmente utilizados na avaliação corporal em relação a factores de risco para a saúde. A estimativa da estrutura óssea pelo valor de perímetro do punho e estatura é de grande utilidade em estudos em que não é possível a utilização de outro método de classificação ${ }^{3,10,13}$. Foram objectivos deste estudo a identificação do estrato social, a avaliação da composição corporal, actividade física e gasto energético assim como o conhecimento das necessidades de hidratação e o estudo do consumo das bebidas no decurso da actividade física. 


\section{METODOLOGIA}

\section{Amostra}

Para selecção da amostra foi estabelecido como critério de inclusão alunos matriculados do $1 \stackrel{0}{0}$ ao $4^{\circ}$ ano que frequentam as aulas da Licenciatura de Ciências do Desporto e Educação Física da Faculdade de Ciências do Desporto e Educação Física da Universidade do Porto, de Março a Maio de 2005. Definiu-se como critérios de exclusão alunos que não preencham os requisitos anteriores descritos. A tabela 1 apresenta o $\mathrm{n}$ ㅇ de alunos avaliados por ano de frequência na Faculdade.

Apesar de este projecto incluir apenas informação observacional, todos os estudantes foram convidados a participar neste estudo e informados sobre os procedimentos utilizados e concordaram em participar voluntariamente no estudo, assinando um termo de consentimento informado da protecção da privacidade de acordo com a Declaração de Helsínquia 1975.

\section{Procedimentos}

Os alunos seleccionados foram avaliados através de parâmetros antropométricos e inquérito específico.

Para a avaliação da composição corporal foi medido; peso (balança Krups), estatura (estadiómetro], perímetro da anca e da cinta (fita métrica flexível]: as pregas cutâneas (lipocalibrador, Holtain), tricipital, bicipital, subescapular, suprailíaca, o perímetro médio do braço e o perímetro do punho [fita métrica flexível] do lado não dominante do participante. Usaram-se técnicas internacionais estandardizadas ${ }^{16}$.

Foi aplicado um inquérito piloto para determinar a validade do mesmo em 40 estudantes do $1^{\circ}$ ao $4^{\circ}$ ano (10 em cada ano escolar, 4 do sexo feminino e 6 do masculino] da Licenciatura de Ciências do Desporto e Educação Física.

0 inquérito aplicado na amostra estudada diferiu do piloto na sua forma e simplicidade das questões. Foi preenchido pelos participantes, depois de uma breve apresentação e contextualização do projecto de investigação e incluía; dados pessoais e sociais questões sobre conhecimentos, consumo e contributo das bebidas para a hidratação antes, durante e depois de actividade física e um questionário sobre a prática da actividade física ${ }^{12,22}$ numa semana típica.

A estimativa do gasto calórico semanal (típica) para todas as actividades físicas foi obtida a partir dos dados da classificação do custo energético das actividades físicas humanas, desenvolvida por Ainsworth et al. e o cálculo proposto por Kriska e Caspersen ${ }^{2,14}$.

Foi calculado o Índice de Massa Corporal $\left(\mathrm{kg} / \mathrm{m}^{2}\right)$ e o Índice cintura/ /anca. A massa gorda [\%] através do somatório das pregas cutâneas

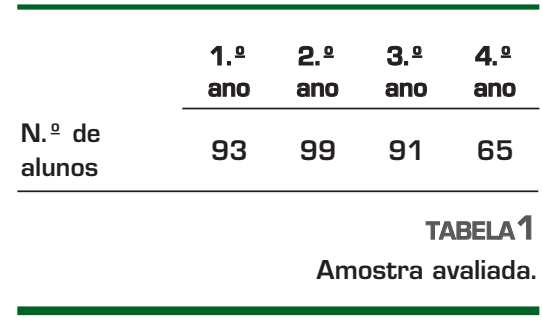

e das formulas de Durnin e Siri e a área muscular do braço $\left(\mathrm{cm}^{2}\right)$ a partir da prega tricipital e o perímetro médio do braço. A classificação de estrutura óssea foi obtida através do valor do perímetro do punho e estatura ${ }^{4,6,10,13}$.

Para caracterização social foi usado a escala de classificação social de Graffar (1- classe alta, 2- classe média alta, 3-classe média, 4- classe média baixa e 5 - classe baixa) ${ }^{11}$.

\section{Análise estatística}

Os dados obtidos foram analisados estatisticamente, pelo Statistical Package for the Social Science [SPSS], versão 13.0 para o Windows e Microsoft Excel 2003.

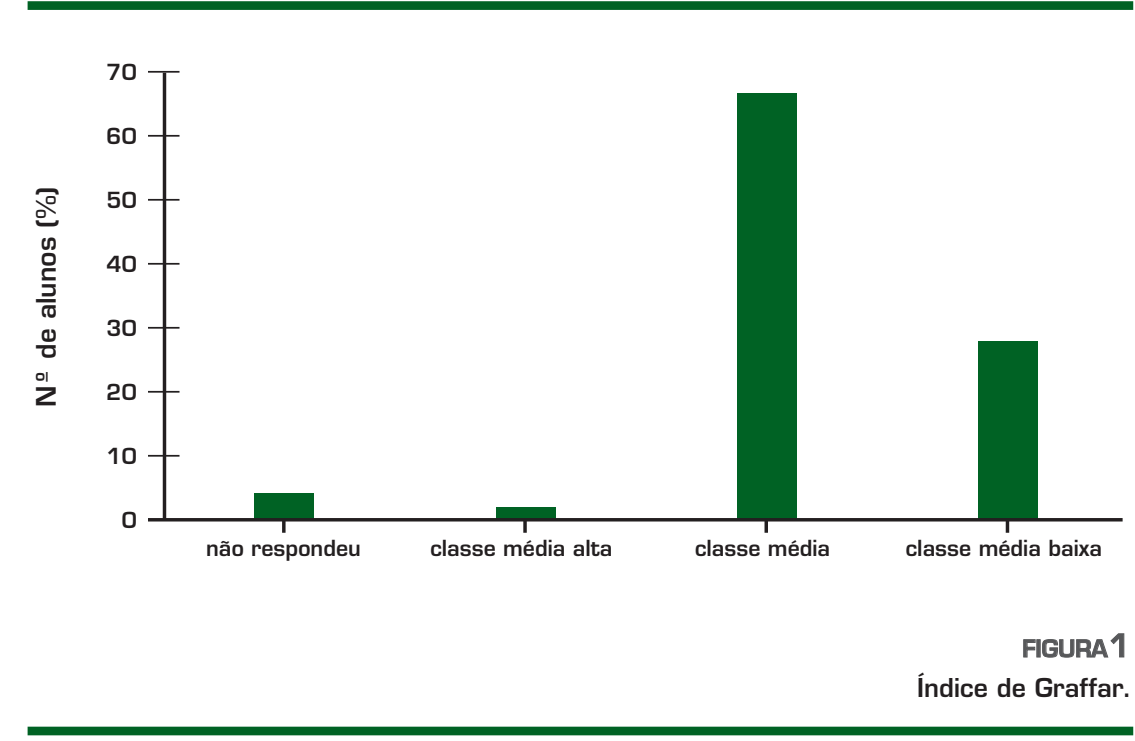




\begin{tabular}{|c|c|c|c|c|}
\hline $\begin{array}{l}\text { Actividades } \\
\text { comuns }\end{array}$ & Intensidade & $\mathbf{N}$ & $\begin{array}{l}\text { Dias } \\
\mathrm{m} \pm \mathrm{dp}\end{array}$ & $\begin{array}{c}\text { Tempo (horas) } \\
\mathrm{m} \pm \mathrm{dp}\end{array}$ \\
\hline \multirow{2}{*}{$\begin{array}{l}\text { Andar a pé } \\
\text { (FCDEF/Emprego) }\end{array}$} & Leve & 183 & $5,1 \pm 1,7$ & $0,66 \pm 0,58$ \\
\hline & Moderado & 49 & $5,7 \pm 1,6$ & $0,96 \pm 1,07$ \\
\hline \multirow{3}{*}{ Caminhada } & Leve & 49 & $2,7 \pm 1,9$ & $0,70 \pm 0,49$ \\
\hline & Moderado & 35 & $2,0 \pm 1,6$ & $1,35 \pm 1,20$ \\
\hline & Vigorosa & 2 & $3,0 \pm 2,8$ & $3,25 \pm 3,89$ \\
\hline \multirow{3}{*}{ Bicicleta } & Leve & 7 & $1,1 \pm 0,4$ & $0,69 \pm 0,41$ \\
\hline & Moderado & 46 & $1,8 \pm 1,1$ & $1,05 \pm 0,75$ \\
\hline & Vigorosa & 9 & $2,0 \pm 1,5$ & $2,75 \pm 2,64$ \\
\hline \multirow{3}{*}{ Andebol } & Leve & 3 & $2,0 \pm 0,0$ & $1,33 \pm 0,58$ \\
\hline & Moderado & 54 & $2,2 \pm 0,8$ & $1,27 \pm 0,63$ \\
\hline & Vigorosa & 34 & $2,8 \pm 1,9$ & $1,77 \pm 1,38$ \\
\hline \multirow{3}{*}{ Atletismo } & Leve & 2 & $1,0 \pm 0,0$ & $1,25 \pm 0,35$ \\
\hline & Moderado & 87 & $1,1 \pm 0,4$ & $1,74 \pm 0,43$ \\
\hline & Vigorosa & 90 & $1,4 \pm 1,2$ & $1,99 \pm 1,08$ \\
\hline \multirow{3}{*}{ Basquetebol } & Leve & 6 & $2,0 \pm 0,0$ & $1,06 \pm 0,47$ \\
\hline & Moderado & 47 & $2,1 \pm 1,1$ & $1,23 \pm 0,56$ \\
\hline & Vigorosa & 32 & $2,8 \pm 1,6$ & $1,50 \pm 1,97$ \\
\hline \multirow{3}{*}{ Futebol } & Leve & 5 & $1,0 \pm 0,0$ & $1,73 \pm 0,44$ \\
\hline & Moderado & 64 & $1,5 \pm 1,0$ & $1,70 \pm 0,60$ \\
\hline & Vigorosa & 89 & $2,4 \pm 1,6$ & $2,00 \pm 1,46$ \\
\hline \multirow{3}{*}{ Ginástica } & Leve & 36 & $2,1 \pm 0,3$ & $1,35 \pm 0,65$ \\
\hline & Moderado & 99 & $2,1 \pm 0,8$ & $1,27 \pm 0,75$ \\
\hline & Vigorosa & 25 & $2,4 \pm 1,7$ & $1,64 \pm 1,26$ \\
\hline \multirow{3}{*}{ Judo } & Leve & 9 & $1,0 \pm 0,0$ & $0,94 \pm 0,08$ \\
\hline & Moderado & 35 & $1,0 \pm 0,2$ & $0,98 \pm 0,30$ \\
\hline & Vigorosa & 17 & $1,4 \pm 1,0$ & $1,39 \pm 1,29$ \\
\hline \multirow{2}{*}{ Natação } & Leve & 27 & $2,1 \pm 0,9$ & $1,07 \pm 0,61$ \\
\hline & Moderado & 72 & $2,3 \pm 1,1$ & $0,97 \pm 0,61$ \\
\hline \multirow{3}{*}{ Voleibol } & Leve & 13 & $1,9 \pm 0,3$ & 1,00 $\pm 0,31$ \\
\hline & Moderado & 64 & $2,0 \pm 1,0$ & $1,45 \pm 1,97$ \\
\hline & Vigorosa & 15 & $2,6 \pm 2,0$ & $2,43 \pm 1,94$ \\
\hline Ex.: Secretária & Leve & 61 & $5,0 \pm 1,8$ & $2,36 \pm 2,02$ \\
\hline \multirow{3}{*}{$\begin{array}{l}\text { Ex.: } \\
\text { Objectos pesados }\end{array}$} & Leve & 8 & $2,5 \pm 1,7$ & $0,50 \pm 0,32$ \\
\hline & Moderado & 30 & $2,9 \pm 2,0$ & $1,45 \pm 1,97$ \\
\hline & Vigorosa & 8 & $2,6 \pm 0,7$ & $1,27 \pm 1,94$ \\
\hline \multirow{2}{*}{ Ex.: Cozinhar } & Leve & 87 & $4,6 \pm 2,0$ & $0,99 \pm 0,38$ \\
\hline & Moderado & 16 & $4,1 \pm 2,6$ & $1,10 \pm 0,96$ \\
\hline \multirow{3}{*}{$\begin{array}{l}\text { Ex.: } \\
\text { Compras pesadas }\end{array}$} & Leve & 45 & $1,5 \pm 1,0$ & $0,40 \pm 0,48$ \\
\hline & Moderado & 26 & $2,1 \pm 1,4$ & $0,48 \pm 0,52$ \\
\hline & Vigorosa & 2 & $1,5 \pm 0,2$ & $0,46 \pm 0,29$ \\
\hline
\end{tabular}

TABELA?

Principais actividades mencionadas pela amostra.

$\mathbf{m} \pm \mathbf{d p}$

média \pm desvio padrão
Além de uma análise estatística descritiva, usou-se a prova de Levene, de Kolmonogorov-Smirnov, de Mann-Whitney, o coeficiente de correlação Spearman e a do Qui-quadrado. Assumiu-se significado estatístico quando $\mathrm{p}<0,05$.

\section{RESULTADOS}

A amostra recolhida representa cerca de $50 \%$ dos alunos matriculados do $1^{\circ}$ ao $4^{\circ}$ ano de Licenciatura de Ciências do Desporto e Educação Física da FCDEF-UP. Dos 355 alunos que reuniam os critérios de inclusão, 5 recusaram participar no estudo e 2 recusaram a avaliação da composição corporal (3ำ ano). A presente amostra compreende 348 alunos $198,0 \%$ da amostra), sendo 93 do $1 \stackrel{\circ}{\circ}$ ano, 99 do $2^{\circ}$ ano, 91 do $3^{\circ}$ ano e 65 do $4^{\circ}$ ano, 140 do sexo feminino e 208 do sexo masculino com a média de idades de 21,0 $\pm 2,8 \mathrm{~cm} \pm$ dp). Pertencentes na sua maioria à classe social média $(66,8 \%)$ estando representados na figura 1.

Dos 348 participantes no estudo, 285 praticam pelo menos uma modalidade desportiva um dia por semana, sendo 89 do $1^{\circ}$ ano, 94 do $2^{\circ}$ ano, 59 do $3^{\circ}$ ano e 43 do $4^{\circ}$ ano. A prática desportiva é igual em todas as classes sociais.

As actividades maioritariamente praticadas pelos alunos foram ginástica, atletismo e futebol. A tabela 2 apresenta as principais actividades físicas (intensidade, dias e horas] mencionadas pelos alunos numa semana típica.

Devido ao reduzido número de alunos de algumas actividades físicas não se efectuou uma análise descritiva. 
A duração das modalidades desportivas foi de aproximadamente duas horas/semana. Dos alunos participantes no estudo, 128 praticam uma modalidade desportiva extracurricular mais de 3 dias/semana e de intensidade elevada.

Dos alunos do Curso de Licenciatura em Ciências do Desporto e Educação Física que praticam modalidades desportivas verificou-se que: 80 de voleibol frequentam o $2^{\circ}$ ano, 80 de futebol o 2 o ano, 84 de andebol o $2^{\circ}$ ano, 86 e 78 de atletismo frequentam o 2 ㅇ e $1 \stackrel{0}{\circ}$ ano respectivamente, 80 e 73 de ginástica o $2^{\circ}$ e $1^{\circ}$ ano, 75 de natação o $1^{\circ}$ ano, 72 de Basquetebol o $1^{\circ}$ ano e 58 de Judo

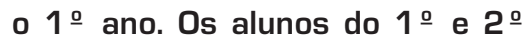
ano praticam mais actividade física do que os do $3^{\circ}$ e $4^{\circ}$ ano.

Na tabela 3, estão apresentados os valores médios $(\mathrm{m} \pm \mathrm{dp})$ de gasto energético com actividade física de 326 alunos. Foram excluídos os valores de GE (gasto energético) igual a zero (15 sexo masculino e 7 sexo feminino].

Encontraram-se correlações moderadas positivas com significado estatístico $(p<0,001)$ entre o gasto energético e a prática (dias e horas] de atletismo, ginástica, judo, andebol, basquetebol, futebol, natação e voleibol.

Os valores médios dos parâmetros da composição corporal apresentam diferenças significativas entre os sexos, mas dentro dos valores esperados para a idade. A tabela 4 apresenta os principais dados $(\mathrm{m} \pm$ dp) da composição corporal.

O IMC apresenta diferenças significativas entre os grupos, mas, em média, corresponde à normoponderabilidade. Os valores médios de massa gorda e área muscular do braço encontram-se dentro dos padrões estabelecidos para a idade e sexo. Os valores médios de estru-

\begin{tabular}{|c|c|c|c|c|c|}
\hline $\begin{array}{l}\text { Ano de } \\
\text { frequência }\end{array}$ & \multicolumn{2}{|c|}{$\begin{array}{c}\text { Sexo } \\
\text { feminino }\end{array}$} & \multicolumn{2}{|c|}{$\begin{array}{c}\text { Sexo } \\
\text { masculino }\end{array}$} & $\mathbf{p}$ \\
\hline $\begin{array}{l}\text { 1/2 GE } \\
\text { (Kcal/semana) }\end{array}$ & \multicolumn{2}{|c|}{$\begin{array}{l}7592,4 \pm 3177,7 \\
3989,3 \pm 2903,0\end{array}$} & \multicolumn{2}{|c|}{$\begin{array}{c}9042,5 \pm 4881,9^{*} \\
6310,6 \pm 3672,0^{*}\end{array}$} & $\begin{array}{r}0,002 \\
<001\end{array}$ \\
\hline [Kcal/semana] & \multicolumn{5}{|c|}{$\begin{array}{l}\text { Caracterização do gasto energético com actividade física da amostra. } \\
\qquad{ }^{*} \mathrm{p}<0,05 ;{ }^{*} \mathrm{p}<0,01\end{array}$} \\
\hline Género & $\begin{array}{c}\text { IMC } \\
{[\mathrm{kg} / \mathrm{m} 2)}\end{array}$ & $\begin{array}{l}\text { MG } \\
{[\%]}\end{array}$ & $\begin{array}{l}\text { AMB } \\
\left(\mathrm{cm}^{2}\right)\end{array}$ & EO & ICA \\
\hline Sexo masculino & $23,9 \pm 2,8$ & $14,8 \pm 3,5$ & $51,1 \pm 11,5$ & $10,2 \pm 0,6$ & $0,8 \pm 0,8$ \\
\hline Sexo feminino & $22,0 \pm 1,9$ & $23,6 \pm 3,6$ & $36,0 \pm 7,8$ & $10,3 \pm 0,4$ & $0,7 \pm 0,0$ \\
\hline
\end{tabular}

TABELA4

Caracterização da composição corporal da amostra.

IMC - Índice de Massa Corporal; MG - Massa Gorda; AMB - Área Muscular do Braço; EO - Estrutura Óssea; * $p<0,05$

LEGENDA

䨽 Aumenta desempenho

$\square$ Diminui sensação de fadiga

\idratação

Amb. quente e hum. elevada

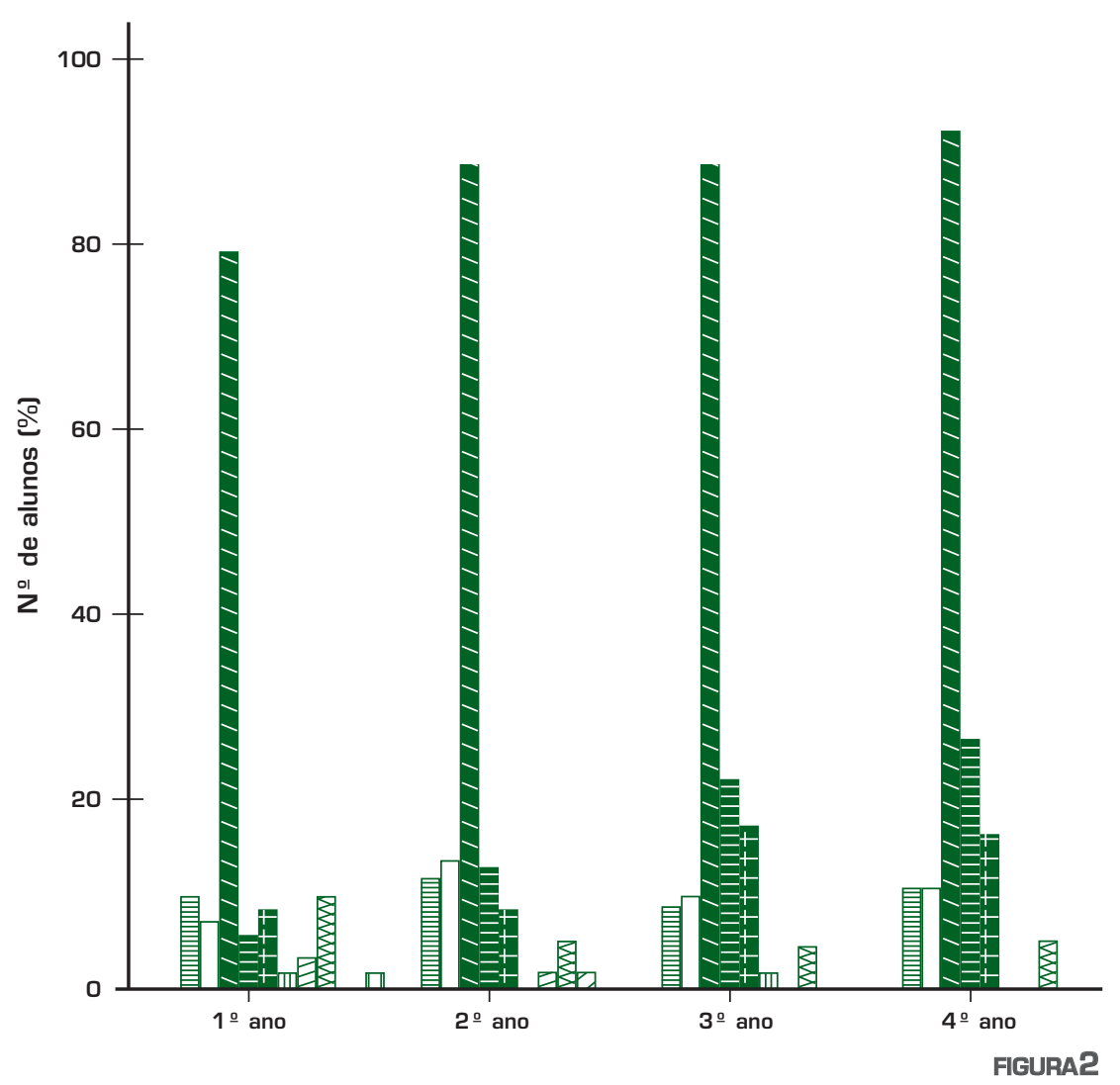

Importância da necessidade de consumo versus não consumo de bebidas antes da actividade física. 


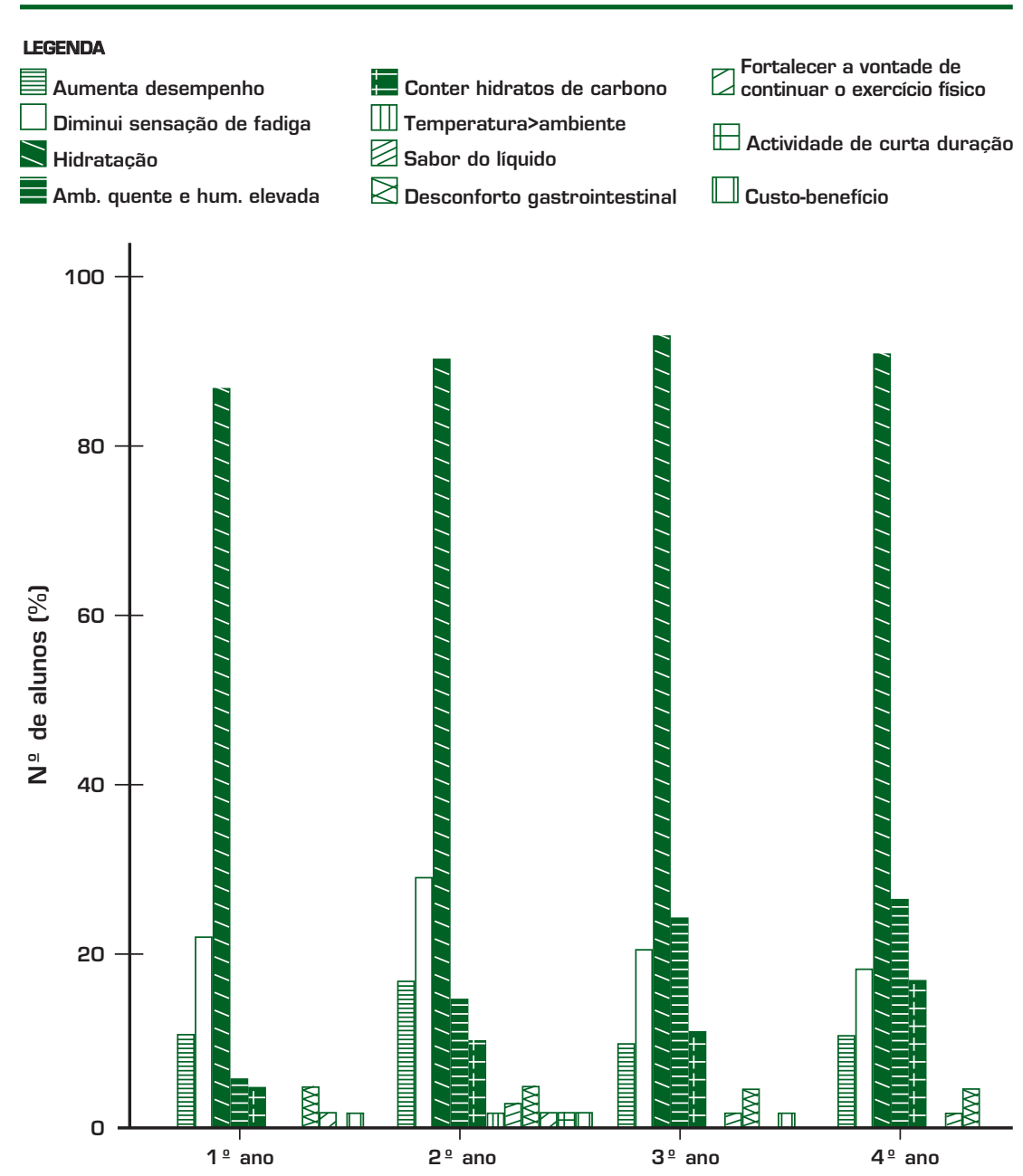

FIGURA3

Importância da necessidade de consumo versus não consumo de bebidas durante a actividade física.

tura óssea para ambos os sexos incluem-se nos intervalos de estrutura intermédia. Os valores médios do Índice de cintura/anca situam-se dentro do recomendado ${ }^{3,13}$.

Verificaram-se correlações ( $p$ - coeficiente de correlação de Spearman] com significado estatístico entre o gasto energético e IMC ( $p=0,237$; $p<0,001)$ e $A M B(p=0,162 ; p=$ 0,002), bem como entre o IMC e AMB $(p=0,564 ; p<0,001)$. Verificou-se uma correlação negativa entre a ICA e EO ( $p=-0,190 ; p<0,001)$ e AMB com MG ( $p=-0,400 ; p<0,001)$.
Existe dependência entre o ano de frequência e a justificação ambiente quente e humidade elevada para o consumo de bebidas antes da actividade física $(p<0,001)$. Tendo os alunos do $3^{\circ}$ e 4 ㅇ ano frequência superior à esperada.

Encontrou-se significado estatístico nas distribuições de frequências de bebidas consumidas antes da actividade física com a menção hidratação $(p=0,007)$, como justificativa dos alunos para o consumo de bebidas antes da actividade física.

Quando se comparam as distribuições de frequências e a menção sabor do líquido $(p=0,026)$ como justificativa para a não necessidade de consumo de bebidas antes da actividade física verificou-se dependência entre as variáveis em estudo.

A figura 3 apresenta as principais justificações dos alunos referentes à necessidade de consumo ou não (temperatura>ambiente, sabor do líquido, desconforto gastrointestinal, fortalecer a vontade de continuar o exercício, actividade de curta duração, custo-benefício] de bebidas durante a actividade física, por ano de frequência da licenciatura da FCDEF-UP.

Existe dependência entre o ano de frequência e a justificação ambiente quente e humidade elevada para o consumo de bebidas durante a actividade física $(p=0,002)$, tendo os alunos do $3^{\circ}$ e $4^{\circ}$ ano frequência superior à esperada.

Encontrou-se relação com significado estatístico entre as distribuições das frequências de bebidas consumidas durante a actividade física e a hidratação ( $p<0,001)$, mencionado pelos alunos para fundamentarem a necessidade de consumo de bebidas durante a actividade física. 
A figura 4 mostra as principais respostas dos alunos referentes à necessidade de consumo ou não (temperatura>ambiente, sabor do líquido, desconforto gastrointestinal, fortalecer a vontade de continuar o exercício, actividade de curta duração, custo-benefício] de bebidas depois da actividade física, por ano de frequência da licenciatura da FCDEF-UP.

Encontrou-se significado estatístico entre as distribuições de frequência de bebidas consumidas depois da actividade física e a hidratação $(p=0,034$ ), como fundamento para a necessidade de ingestão de bebidas depois da actividade física. Dos resultados obtidos anteriormente [com nível de significância estatística] pode-se afirmar que os alunos que consideram importante o consumo de bebidas antes, durante e depois de actividade física devido às necessidades de hidratação ingerem mais bebidas antes, durante e depois da actividade física respectivamente. Os alunos do $3^{\circ}$ e $4^{\circ}$ ano mencionaram mais ambiente quente e humidade elevada como fundamento da necessidade de consumo de bebidas antes e durante actividade física.

O sabor do liquido foi a menção dos alunos mais frequente para a não necessidade de consumo de bebidas antes da actividade física. Contudo para as restantes mencões justificativas para o consumo ou não, verificou-se independência entre as variáveis.

Dos alunos participantes, 304 consideram que as necessidades de hidratação variam com a modalidade desportiva. A figura 5 apresenta as principais menções dos alunos para as necessidades de hidratação ou não (duração do exercício, necessidades individuais, ambiente quente e humidade eleva-

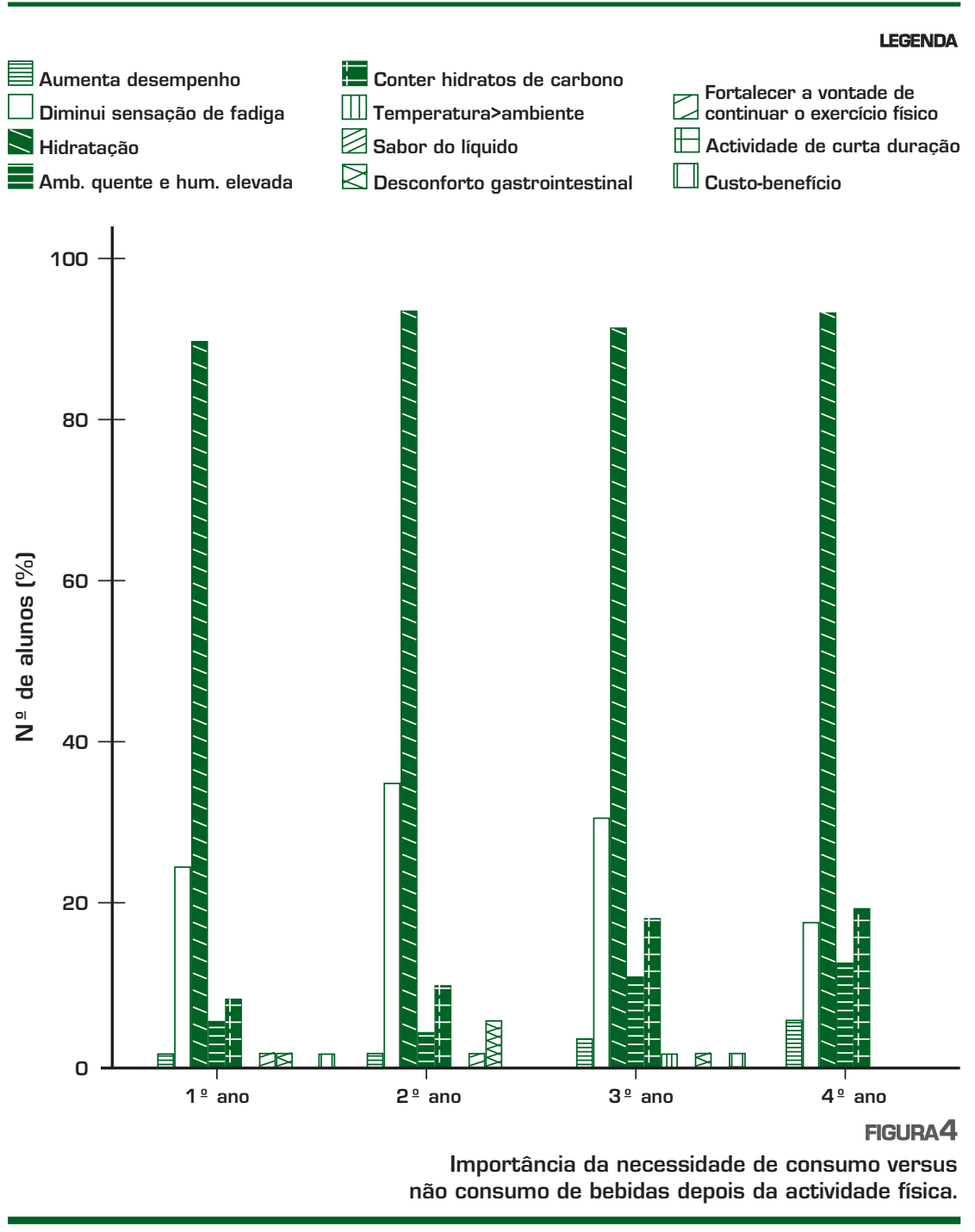

da e quantidade de suor] consoante a modalidade desportiva, por ano de frequência da licenciatura da FCDEF-UP.

Quando se compara por ano de frequência verifica-se que existe dependência com a menção diferentes meios ambientais referida pelos alunos, tendo o $1 \stackrel{0}{ }$ e 20 ano valores observados superiores aos esperados.

Dos alunos que praticam actividade física, $85,6 \%, 78,6 \%$ e $89,8 \%$ consomem bebidas antes, durante e depois da prática de pelo menos uma modalidade desportiva respectivamente. A figura 6 apresenta o consumo de bebidas no decurso da actividade física por ano de frequência da Licenciatura de Ciências do Desporto e Educação Física. Verificou-se dependência das frequências entre os alunos que ingerem líquidos antes da actividade física e o ano de frequência, tendo-se observado para o 2 o ano valores superiores ao esperado. 0 consumo de bebidas antes, durante $\mathrm{e}$ depois é independente da classe social do aluno. 

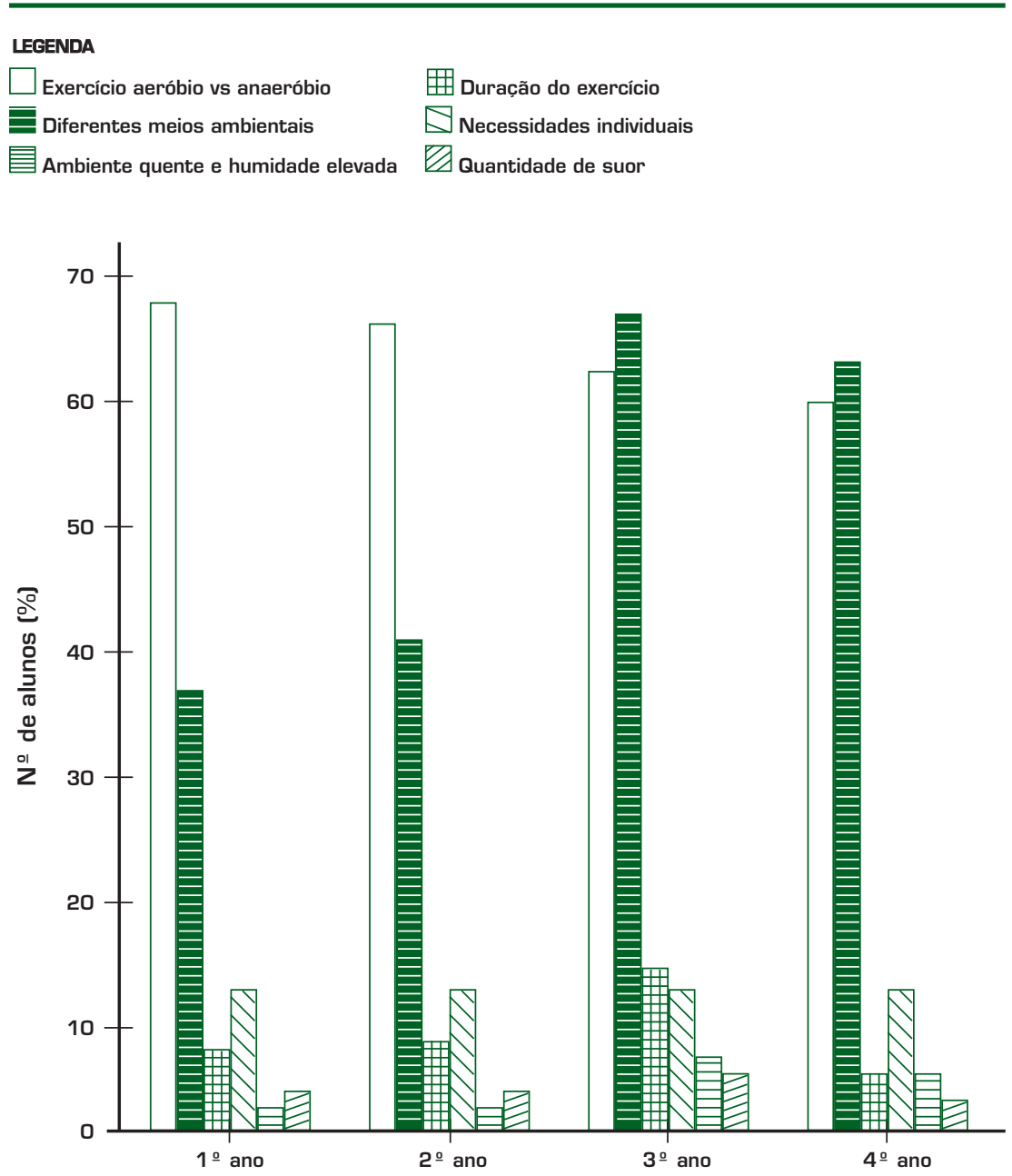

FIGURA5

Principais menções das necessidades de

hidratação consoante a modalidade desportiva.

As tabelas 5, 6, 7 apresentam as principais bebidas ingeridas pelos alunos $(n / N)$ antes, durante e depois das modalidades desportivas (MD) mais praticadas pelos alunos participantes, respectivamente.

Dos 128 alunos que praticam modalidades desportivas extracurriculares mais de $\mathbf{3}$ dias/semana de intensidade elevada, 2 não ingerem líquidos no decurso da actividade física, 20 antes, 29 durante e 16 depois da actividade física, sendo a água a bebida seleccionada pela maioria $[86,5 \%)$ dos alunos.
Encontrou-se correlações moderadas e positivas com significado estatístico $(p<0,001)$ entre o $n \stackrel{0}{0}$ de dias/semana das modalidades praticadas pelos alunos participantes e o $\mathrm{n}^{\circ}$ de dias/semana que consomem bebidas antes, durante e depois das actividades físicas.

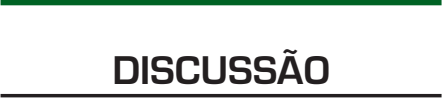

Os alunos avaliados praticam na sua maioria voleibol, atletismo, ginástica, basquetebol, natação, andebol, judo e futebol contribuindo positivamente para o gasto energético com a prática desportiva. Sendo estas modalidades integradas nas disciplinas curriculares da Licenciatura em Ciências do Desporto e Educação Física.

Neste estudo encontrou-se diferenças significativas entre os sexos quanto à composição corporal como de acordo com outros estudos. Os valores da composição corporal encontram-se dentro do recomendado para idade e sexo, o que seria de esperar, sendo a amostra constituída por alunos fisicamente activos ${ }^{10}$. Uma actividade física intensa e prolongada leva a um aumento significativo da massa muscular o que implica um aumento de peso e consequentemente do IMC. Neste estudo os valores de IMC da amostra correlacionaram-se positivamente com a área muscular do braço e o gasto energético despendido na actividade física. Verificou-se também que quanto maior a área muscular do braço menor a percentagem de massa gorda corporal dos alunos. Um nível adequado de hidratação só é mantido em pessoas fisicamente activas se beberem líquidos suficientes antes, durante e depois de actividade física com duração superior a uma hora ${ }^{1,7}$. A necessidade de hidratação foi a justificação dos alunos com maior frequência para o consumo de bebidas antes, durante e após actividade física. Verificou-se que estes alunos têm uma maior tendência de ingerir líquidos antes, durante e depois actividade física.

Febbraio et al. ${ }^{8}$ demonstraram que a ingestão de hidratos de carbono antes e durante a actividade física acrescenta um efeito adicional no desempenho quando comparado com os hidratos de carbono ingeridos ou antes ou durante a actividade física. A adição de hidratos 
de carbono a uma solução de hidratação acelera o processo de recuperação depois de actividade física ${ }^{1,8}$. Alguns alunos referiram a importância da adição de hidratos de carbono nas bebidas consumidas antes, durante e depois da actividade física.

0 mecanismo da sede é insuficiente para repor os niveis hídricos após uma depleção acentuada de água corporal. A ingestão voluntária de líquido é influenciada por uma variedade de informações sensoriais como o odor, gosto, temperatura, cor e qualidade, são todas características que influenciam a palatibilidade e por isso tendem a estimular o consumo de líquidos durante a actividade física. Apenas poucos factores têm sido estudados, os principais foram a temperatura e o sabor do líquido ${ }^{4,7,15}$. Foi mencionado o sabor do líquido como justificação da não necessidade de consumo de bebidas antes da actividade física. A perda de líquidos e sais minerais durante a actividade física depende não só da sua duração e intensidade, mas também das condições climáticas. $\mathbf{0}$ suor varia individualmente sendo influenciado pela intensidade da actividade física, o estado de adaptação e as condições ambientais. Em ambientes quentes e húmidos aumenta a taxa de eliminação do calor e a humidade diminui a eficiência da evaporação; em alguns casos o frio pode desidratar de forma severa ${ }^{4,7,19} .0$ ambiente quente e humidade elevada foram das menções referidas com significado estatístico relevante entre alunos que ingerem líquidos antes e durante a actividade física e superior ao esperado para os alunos do $3^{\circ}$ e $4^{\circ}$ ano da FCDEF-UP. Podendo este resultado obtido ser devido ao maior conhecimento dos

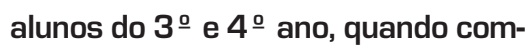
parado com os do $1^{\circ}$ e $2^{\circ}$ ano.

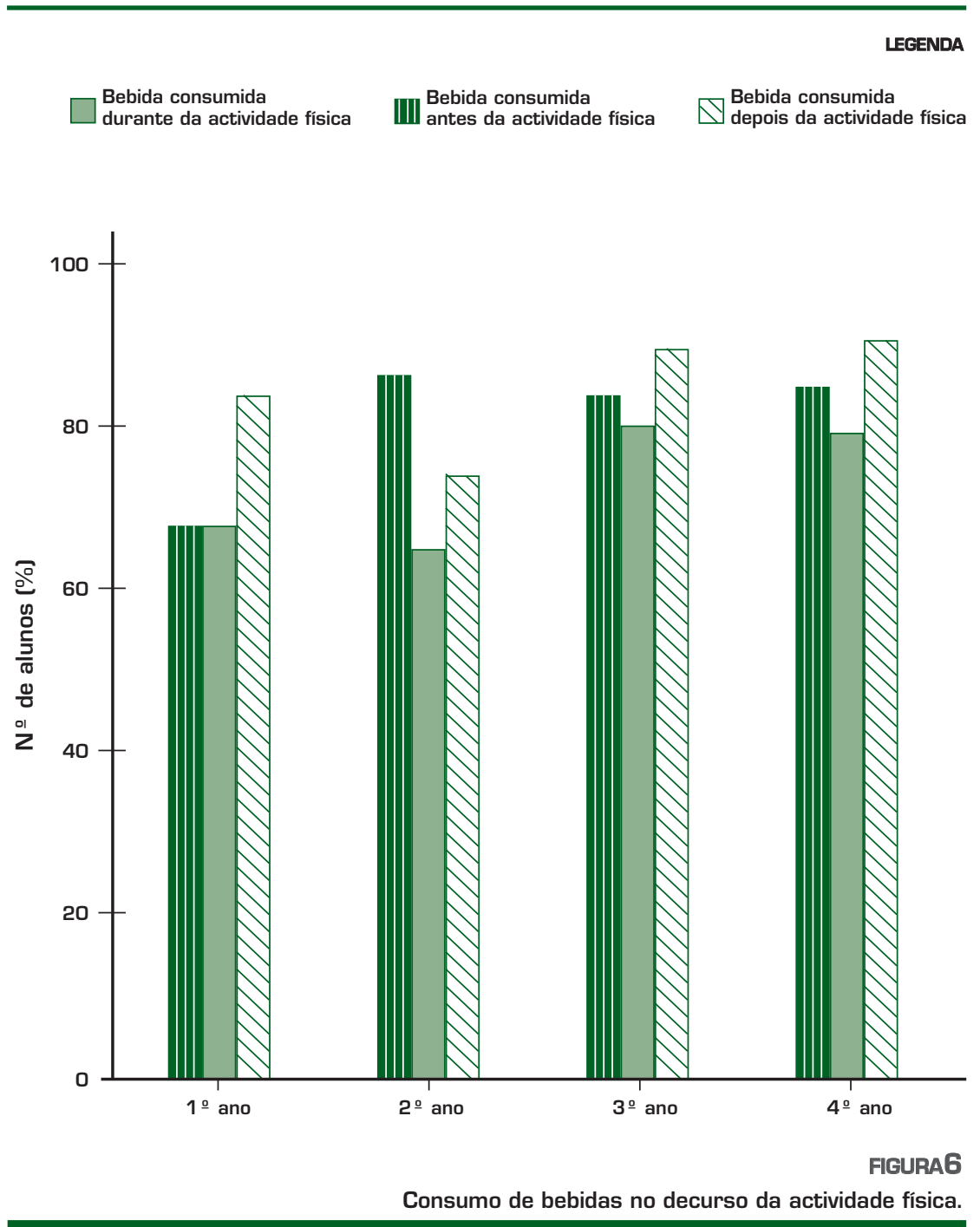

A questão da hidratação, seja antes, durante ou depois da realização da actividade física, independente de ser aeróbia ou anaeróbia, tem grande importância para o bom funcionamento dos processos homeostáticos exigidos pela actividade física. No entanto, o exercício aeróbio prolongado é mais afectado negativamente pela hipohidratação do que o exercício anaeróbio de curta duração ${ }^{7,19}$. Neste estudo os alunos consideram que as necessidades de hidratação variam com o tipo de actividade física.
Um reduzido número de alunos avaliados neste estudo consumiram bebidas antes, durante $\mathrm{e}$ depois da actividade física, sendo menos frequente nas modalidades desportivas curriculares. As modalidades desportivas em que os alunos consumiram mais bebidas foram no Futebol e no Andebol e menos o Judo. Sendo de salientar que o Andebol e Futebol são as modalidades desportivas extracurriculares mais praticadas pelos alunos ao contrário do Judo. 


\begin{tabular}{|c|c|c|c|c|c|c|}
\hline $\begin{array}{l}\text { Modalidades } \\
\text { Desportivas } \\
\text { [n] }\end{array}$ & $\begin{array}{l}\text { Água } \\
\text { [n] }\end{array}$ & $\begin{array}{c}\text { Água ou } \\
\text { isotónico } \\
\text { [n] }\end{array}$ & $\begin{array}{l}\text { Água ou } \\
\text { sumo } \\
\text { [n] }\end{array}$ & $\begin{array}{c}\text { Sumo } \\
\text { [n] }\end{array}$ & $\begin{array}{c}\text { Outros } \\
\text { [n] }\end{array}$ & $\begin{array}{l}\text { N (\%) que pratica } \\
\text { MD e ingere liquidos } \\
\text { [n] }\end{array}$ \\
\hline Voleibol (40) & 34 & 2 & 1 & 0 & 3 & 43,5 \\
\hline Atletismo [86] & 69 & 1 & 6 & 6 & 4 & 48,0 \\
\hline Andebol (50) & 35 & 5 & 1 & 3 & 6 & 54,9 \\
\hline Judo [22] & 11 & 0 & 0 & 0 & 12 & 36,1 \\
\hline Ginástica [51] & 43 & 1 & 2 & 1 & 4 & 31,9 \\
\hline Natação [34] & 24 & 0 & 0 & 4 & 6 & 34,3 \\
\hline Basquetebol (28) & 20 & 2 & 0 & 0 & 6 & 32,9 \\
\hline Futebol (95) & 81 & 2 & 1 & 1 & 10 & 60,1 \\
\hline
\end{tabular}

TABELA5

Bebidas consumidas antes da prática desportiva.

\begin{tabular}{|c|c|c|c|c|c|c|}
\hline $\begin{array}{l}\text { Modalidades } \\
\text { Desportivas } \\
\text { [n] }\end{array}$ & $\begin{array}{c}\text { Água } \\
\text { [n] }\end{array}$ & $\begin{array}{l}\text { Água ou } \\
\text { isotónico } \\
\text { [n] }\end{array}$ & $\begin{array}{l}\text { Água ou } \\
\text { sumo } \\
\text { [n] }\end{array}$ & $\begin{array}{c}\text { Sumo } \\
\text { [n] }\end{array}$ & $\begin{array}{c}\text { Outros } \\
\text { [n] }\end{array}$ & $\begin{array}{l}\text { N (\%) que pratica } \\
\text { MD e ingere líquidos } \\
{[n]}\end{array}$ \\
\hline Voleibol [31] & 29 & 1 & 1 & 0 & 0 & 33,7 \\
\hline Atletismo [66] & 62 & 0 & 1 & 3 & 0 & 36,9 \\
\hline Andebol [43] & 28 & 0 & 0 & 1 & 4 & 47,3 \\
\hline Judo [9] & 9 & 0 & 0 & 0 & 0 & 14,8 \\
\hline Ginástica [33] & 28 & 3 & 0 & 2 & 0 & 20,6 \\
\hline Natação (25) & 21 & 2 & 1 & 0 & 1 & 25,3 \\
\hline Basquetebol (28) & 25 & 2 & 0 & 0 & 1 & 32,9 \\
\hline Futebol (85) & 82 & 0 & 2 & 1 & 0 & 53,8 \\
\hline
\end{tabular}

TABELA6

Bebidas consumidas durante a prática desportiva.

\begin{tabular}{|c|c|c|c|c|c|c|}
\hline $\begin{array}{l}\text { Modalidades } \\
\text { Desportivas } \\
\text { [n] }\end{array}$ & $\begin{array}{c}\text { Água } \\
\text { (n) }\end{array}$ & $\begin{array}{c}\text { Água ou } \\
\text { isotónico } \\
\text { [n] }\end{array}$ & $\begin{array}{l}\text { Água ou } \\
\text { sumo } \\
\text { [n] }\end{array}$ & $\begin{array}{c}\text { Sumo } \\
\text { [n] }\end{array}$ & $\begin{array}{l}\text { Outros } \\
\text { [n] }\end{array}$ & $\begin{array}{l}\text { N (\%) que pratica } \\
\text { MD e ingere líquidos } \\
\text { [n] }\end{array}$ \\
\hline Voleibol [46] & 36 & 0 & 7 & 1 & 2 & 50,0 \\
\hline Atletismo [85] & 68 & 0 & 10 & 2 & 5 & 47,5 \\
\hline Andebol [51] & 40 & 3 & 0 & 2 & 6 & 56,0 \\
\hline Judo [16] & 14 & 0 & 1 & 1 & 0 & 26,2 \\
\hline Ginástica [51] & 43 & 0 & 0 & 2 & 6 & 31,9 \\
\hline Natação (43) & 34 & 1 & 2 & 2 & 4 & 43,4 \\
\hline Basquetebol (42) & 35 & 1 & 0 & 6 & 0 & 49,4 \\
\hline Futebol [99] & 83 & 1 & 12 & 3 & 0 & 62,7 \\
\hline
\end{tabular}

TABELA7

Bebidas consumidas depois da prática desportiva.
Directamente associadas a actividades físicas, as bebidas desportivas viabilizam a rápida absorção de sais e de água perdidos pelo organismo através da transpiração, necessária para o equilíbrio da temperatura corporal ${ }^{1,7}$. A eficácia fisiológica requer que a bebida formulada evite as limitações impostas pela ingestão voluntária, esvaziamento gástrico e absorção intestinal, ao mesmo tempo fornecendo líquido, hidratos de carbono e electrólitos em quantidades e frequências conhecidas por provocar respostas fisiológicas e de desempenho $^{18,19,21}$. Mas representam um elevado custo, para obtenção de hidratos de carbono e líquidos. Dos alunos que praticam actividade física e ingerem líquido antes, durante e após a actividade física, as bebidas desportivas foram menos consumidas e as energéticas não foram seleccionadas por nenhum aluno.

A bebida mais consumida antes, durante e depois da actividade física foi a água seguida de sumo. Para a maioria das formas de desenvolvimento da actividade física moderadas, não são necessários líquidos especiais para a hidratação. Para indivíduos em treino e competição basta estimular a ingestão de água na actividade física, líquidos adequados são necessários para aqueles que praticam actividades de elevada intensidade ${ }^{1,7,17}$. A maioria dos alunos participantes no estudo praticam modalidades desportivas de intensidade moderada. No entanto, os alunos que praticam modalidades desportivas extracurriculares intensas preferem mais consumir água antes, durante e depois da actividade física. A referência de alguns alunos quanto à prática de actividade física intensa nas modalidades desportivas curriculares possivelmente deve-se ao grande esforço físico que neces- 
sitam de despender e que não o fazem habitualmente. Sendo de evidenciar que é mais frequente em alunos que não praticam modalidades extracurriculares. A sensação de esforço é assim determinada pelas experiências anteriores.

A bebida que os alunos participantes no estudo mais ingerem durante a actividade física com a adição de hidratos de carbono é o sumo (Cem gramas contem cerca de 85 a $91 \mathrm{~g}$ de água, 9 a $15 \mathrm{~g}$ de hidratos de carbono, 5 a $10 \mathrm{mg}$ de sódio, 60 a $80 \mathrm{mg}$ de potássio e 3 a $2 \mathrm{mg}$ de magnésio). Alguns sumos de fruta (kiwi, maçã, figos, pêra, abacaxi] podem conter quantidades significativas de frutose quando comparada com os outros hidratos de carbono presentes nas bebidas. As quantidades de frutose nos sumos poderão ser contraindicadas, uma vez que a frutose terá que primeiro ser convertida em glicose no fígado antes de ser metabolizada pelos músculos, tornando portanto a frutose menos efectiva como fonte principal de energia para o desempenho atlético. Indivíduos que participaram em pesquisas nas quais foi oferecido somente solução de frutose para hidratação reclamaram desta, pois apresentavam mal-estar gastrointestinal, diarreia e vómito. Sendo de salientar que o uso de bebidas contendo múltiplos de hidratos de carbono, estimula os diferentes mecanismos de absorção do soluto, resultando numa maior absorção de água se comparada a bebidas com apenas um tipo de hidratos de carbono ${ }^{5,9,20}$.

A água foi a bebida mais consumida pelos alunos no decurso da actividade física moderada, intensa e prolongada. Em situações de trabalho moderado, não é necessária a reposição de minerais, visto que pela alimentação normal os níveis são restabelecidos. No entanto, em actividade física intensa e prolongada é aconselhável a ingestão de líquidos que contenham quantidades adequadas de sais minerais. Quando comparamos modalidades desportivas verifica-se que algumas não incluem condições e oportunidades frequentes e/ou para ingerir volume adequado de líquidos e assim prevenir a desidratação. Estudos relevam que a ingestão de líquidos aromatizados e com adição de sódio estimulam a vontade de beber e consequentemente ao aumento da sensação de sede quando comparado com a água pura, ${ }^{5,15}$. Sendo de salientar que as práticas desportivas mais praticadas pela amostra estão incluídos nas disciplinas curriculares da Licenciatura de Ciências do Desporto e Educação Física e que a ingestão de líquido pode ser limitante pelo pouco tempo que têm disponivel da prática de uma modalidade desportiva para a seguinte.

A ingestão de líquidos durante a actividade física é talvez a recomendação mais importante na posição da ACSM, pelo facto de identificar que o objectivo ideal da ingestão é prevenir qualquer nível de desidratação ${ }^{1,4,19}$. Os alunos que praticam as modalidades desportivas analisadas ingerem menos bebidas antes e durante a prática desportiva, observando-se o menor consumo durante a actividade física. No presente estudo quando se comparou o consumo de bebidas no decurso da actividade física, verificou-se que os alunos consomem mais bebidas depois da actividade física.

A hidratação foi para os alunos participantes no estudo a principal justificação para a necessidade e consumo de bebidas antes, durante e depois da actividade física. No entanto, um reduzido número de alunos ingerem bebidas no decurso das modalidades desportivas curriculares. Os alunos fisicamente activos estarão interessados em líquidos que promovam o aumento do desempenho desportivo. Educar os alunos fisicamente activos sobre os efeitos da desidratação no desempenho físico corresponde a informá-los como devem monitorizar os estados de hidratação em função da modalidade desportiva e incentivar técnicas durante os treinos e competicõos.

Este trabalho teve como objectivo principal estudar o consumo e a importância de bebidas que possam de alguma forma apoiar a manutenção do equilíbrio hídrico de no decurso da actividade física em alunos fisicamente activos. Pode-se também considerar que de uma perspectiva geral os objectivos foram alcançados. No entanto, é necessário evidenciar que um estudo em indivíduos fisicamente activos pressupõe uma avaliação mais alargada, na medida que vários factores como as características específicas de cada modalidade desportiva e as necessidades individuais de cada aluno contribuem para alcançar níveis adequados de hidratação, não dependendo assim somente do consumo ou não de bebidas no decurso da actividade física.

\section{AGRADECIMENTOS}

A realização deste trabalho beneficiou do apoio financeiro do Instituto de Bebidas e Saúde - iBeSa, através da concessão de uma bolsa de incentivo à pesquisa e investigação e foi parcialmente apresentado publicamente a 7 de Fevereiro de 2006 na $3^{\text {a }}$ Jornada Bebidas e Saúde na Faculdade de Ciências da Universidade do Porto. 


\author{
CORRESPONDÊNCIA \\ Carla Andreia Lima Ferreira

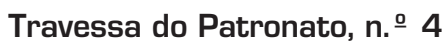 \\ 9060-219 Funchal \\ E-mail: c.carla.ferreira@gmail.com
}

\section{REFERÊNCIAS}

1. American College of Sports Medicine (1996). Position Stand on exercise and Fluid Replacement. Med Sci Sports Exerc 28(1):i-vii.

2. Ainsworth BE, Maskell WL, Whitt MC, Irwin ML, Swartz SJ et al (2000). Compendium of Physical Activities: an update of activity codes and Met Intensities. Med Sci Sports Exerc 32(9): Suppl: 498-516.

3. Bray GA, Gray DS (1988). Anthropometric measurements in the obese. In Lohman TG, Roch AF, Martorelli R. Anthropometric standardization reference manual. Champaign: Human Kinetics, 131-136.

4. Burke LM, Hawley JA (1997). Fluid Balance in Team Sports. Sports Med 24(1):38-54.

5. Chang RT, Gisolfi CV (1995). Effects of carbohydrates type and concentration and solution osmolality on water absorption. Med Sci Sports Exerc 27:1607-1615.

6. Durnin JUVA, Womersley J (1974). Body fat assement from fat total body density and its estimation from skinfold thickness: measurement on 481 men and women 16 to 72 years. British $J$ Nut 32:77-97.
7. Encyclopaedia of Sports Medicine na IOC Medical Commission Publication (volume VII) in colloboration with the International Federation of Sports Medicine (2000) Nutrition in Sport. Oxford: Blackewell Science. 8. Febbraio MA, Chiu A, Augus DJ, Arkinstall MJ, Hawley JA (2000). Effects of carbohydrate ingestion before and during exercise on glucose kinetics and performance. J Appl Phsiol 89:2220-2226.

9. Ferreira FAG, Graça MÊS (1977). Tabela de composição dos alimentos Portugueses. Lisboa: Instituto Ricardo Jorge.

10. Frisanch AR (1990). Anthropometric Standars for the assessment of growth and nutritional status. Ann Arbor, MI: University of Michigan Press.

11. Graffar M. [1996) "Une méthode classification sociale d'echatillons de la population"- Courier 6:455.

12. Craig CL, Marshall AL, Sjöström M, Bamean AE, Booth ML, Ainsworth BE et al (2003). International Physical Activity Questionnaire: 12-Country Reliability and Validity. Med Sci Sport Exerc 35(8):1381-1395.

13. Himes JH (1991). Considering frame size in nutrition assessment. In: Hilmes JH (ed). Anthropometric assessement of nutritional satus. New Work: Wiley -Liss, 141-150.

14. Kriska AM, Caspersen LJ (1997). Introdution to collection of physical activity questionnaires. Med Sci Sport Exerc 29 suppl 6:5-9.

15. Minehan MR, Riley MD, Burke CM (2002). Effect of flavor and awareness of kilojoule content of drinks in preference and fluid balance in team sports. Int $J$ Sport Nutr Exerc Metab 12:81-92.

16. Norton K, Olds T, Australian Sports Commission (1998). Anthropometrica. Sidney: UN SW Press.

17. Position of the American Dietetic Association, Dietitians of Canada, and the American College of Sports Medicine (2000). Nutrition and atletic performance. J Am Diet Assoc 100:1543-56.

18. Serge P, Brauss WA, Markojski MS, Beneke R, Leithauser R [2004). Fluids and Hidration in Prolonged Endurance Performance. Nutrition 20:651-656.

19. Shirreffs SM, Armstrong CE, Cheuvont SM (2004). Fluid and electrolyte need for preparation and recovery from training and competition. J Sports Sci 22:57-63.

20. United States Department of Agriculture. (?) Human Nutrition. Information Service. Sugars contents of Select Foods. Individual and Total Sugars. Home Economics Research Report nㅇ 48.

21. Vinci G, lannilli I, Restuccia D, Santini I, Amendola C (2004). Multivariate Statistical Analysis Companing sport and energy drinks. Innovative Food Sci Emerging Technologies 5:263-267.

22. Weudel-Vos GCW, Schuit AJ, Saris WHH and Kromhout D (2003). Reproducibility and relative validity of the Short Questionnaire to Asses Health-enhancing physical activity. $J$ Clin Epidem 56:1163-1169. 